\title{
Effects of Instability Tools on Muscles Activities in Lunge Exercise in Healthy Adult Males
}

\author{
KwangSu Kim', JaeHong Lee², JinHwan Lee², JaeKwang Lee ${ }^{1}$ \\ 'Department of Physical Therapy, Graduate School, Daegu Catholic University, Daegu; ${ }^{2}$ Department of Physical Therapy, Daegu Health College, \\ Daegu, Korea
}

Purpose: This study was to investigate the effect of instability on the activation of lower limb muscles during lunge exercise.

Methods: The study subjects were healthy twenties and were selected to have no orthopedic or neurological diseases. 1) Flat lunge exercise (Lunge 1, 2) Lunge exercise on TOGU (Lunge 2,3) Holding the olympic bar and moving the lunge on TOGU $($ Lunge 3 , 4) Holding the Surge and moving the lunge on TOGU (Lunge 4). Through the above four actions, we can see how the top-down instability influences the lower limb unlike the Bottom-up instability. EMG attachment sites were gluteus medius, vastus medialis, gastrocnemius, tibialis anterior. Results: These result suggest that exercise using Lunge 4 activity was the highest in muscle activity compared to other exercises, but vastus medialis showed the highest muscle activity in Lunge 2 exercise $(p<0.05)$.

Conclusion: This study showed the muscular activity of the lower extremities according to the lunge exercise using the instability tools.

Keywords: Electromyography, Balance exercise, Lunge exercise, Top-down exercise

\section{INTRODUCTION}

Currently, the public popularity of exercise programs using bottomup-type instability device such as TOGU and BOSU balls has gradually increased, becoming a popular means of exercise. ${ }^{1}$ Exercise using these instability device helps improve the ability of the muscles to contract at the same time by increasing muscle strength due to neural adaptation and activating core muscles, compared to performing similar exercises on a stable ground. ${ }^{2}$ It has also been reported that it can stimulate more neuromuscular than resistance exercises on a stable ground. ${ }^{3}$

Recent studies have highlighted the importance of lower extremity muscles in order to ensure the stability of the body and prevent injury. ${ }^{4}$ Squats and lunge are typical close-kinetic chain exercises, which are effective methods of exercise for the development of lower extremity muscles. ${ }^{5}$ Lunge is a posture that stimulates the mobility and stability of the hips, knees, ankles, and foot. ${ }^{6}$ Also, the lunge requires more left and right movement and balance than the squats,

Received Nov 13, 2019 Revised Dec 21, 2019

Accepted Dec 30, 2019

Corresponding author JaeHong Lee

E-mail heart0630@dhc.ac.kr since weight is applied to the legs on the floor, storing the propulsion in elastic energy, and then converting it back into a thrust that returns to its place. ${ }^{7.8}$ Also, the difficulty is high among close-kinetic chain exercises because lunge exercises require a variety of lower extremity physical abilities, including physical strength and agility, and provide instability on both sides of the left and right than squats exercises. ${ }^{9}$

Devices that provide commonly used instability are bottom-up tools that transmit instability from the lower to the upper body. However, in this study, we want to apply top-down methods that provide instability from the upper body to the lower body. The flexbar, a representative device, is an unstable resistor tool that provides resistance by actively shaking, and has the advantage of alternating contraction of protagonist and antagonist to control instability during exercise on the 3 of the exercise planes (frontal plane, sagittal plane, transverse plane). However, it is an expectable resistance that can determine the direction and intensity of instability on its own. ${ }^{10}$ In contrast, surge puts water into a long plastic cylinder, creating

Copylight (C2019 The Korean Society of Physical Therapy

This is an Open Access article distribute under the terms of the Creative Commons Attribution Non-commercial License (https://creativecommons.org/license/by-nc/4.o.) which permits unrestricted non-commercial use, distribution, and reproduction in any medium, provided the original work is properly cited. 
unexpectable resistance as a hydrodynamic training device that stimulates each part of the body according to the waves, dives, and flows of water. Recommend to some athletes training using surge because it is effective to train in the same environment as the athletic environment. Also, using top-down and bottom-up methods in situation may improve the training system of athletes according to the principle of training specificity. For example, training using BOSU similar to water level to improve surfing skills. In contrast, swing movements in golf or baseball, where upper body rotation is transmitted to lower body instability, are performed on a stable ground with top-down type unstable resistance.

In order to maximize the effect of the lunge, it will be possible to compare the activity of the lower extremity muscles in various environments (stable surface, bottom-up, top-down) to identify the effectively applicable instability devices. Therefore, in this study, would like to compare the differences in activity of the lower extremity muscles during the performance of the 4 exercises using the instability devices with young adults in 20 s, thereby presenting the most effective method of lunge for the purpose.

\section{METHOD}

\section{Participants}

\section{1) Selection criteria of participants}

This study was conducted with 20 healthy adult males in their 20 s living in Daegu. The participants were measured for changes in the activity of the lower extremities by providing various instability during the lunge exercise. The research participants selected received a thorough explanation related to the research objectives and methods before the experiment and voluntarily agreed to participate. This study complied with the ethical standards of the declaration of Helsinki. The criteria for selecting a participants are as follows. First, a person without congenital deformities or orthopedic diseases. Second, a person who has no damage to the central or peripheral nerve. Third, a person with the dominant foot on the right.

\section{2) General characteristics of research participants}

The general characteristics of the participants were 20 normal adult males, with an average age of 22.1 years, an average height of 173.1 $\mathrm{cm}$, an average weight of $71.7 \mathrm{~kg}$.

\section{Procedures}

\section{1) Experimental procedures and tools}

Before the experiment, a briefing was given to the participants to explain the significance of the experiment and then draw up a consent form for participation in the experiment. Later, collected physical characteristics (name, gender, age, height, weight) and participants with dominant right foot carried out lunge exercises during the next exercise. ${ }^{13}$ The participants of the experiment were a total of 20 healthy males, performing the lunge exercises according to their respective conditions, and the sequence of exercises was randomly assigned. Perform it for 10 seconds each time, and a total of 3 times as a set. In order to obtain more clear data, muscle activity measured during the 4 second period was used, except for the first 3 seconds and the last 3 seconds. To minimize the fatigue of the participants, each set was given a 30 -second break and five minutes of rest after exercise.

The method of exercise was divided into Lunge 1-4 depending on the condition of the ground and the weight applied to the upper body. The devices used for this were TOGU for provides instability on the ground, Olympic bar $5.5 \mathrm{~kg}$ for stable weight resistance on the upper body, and surge (Hedstorm Fitness, USA) for instability weight resistance on the upper body, which contained water the same weight as Olympic bar. Surge is a new-concept exercise device that uses water's dynamic movements inside a cylinder as a weight resistance.

The lunge is their legs about the width of their pelvis in a standing position and extends their right leg forward about 70 to $100 \mathrm{~cm}$, and extends back and waist with up left leg heel. Then bend the knee of your right leg 90 degrees and lower yourself to the point where you feel the knee of your left leg touching the floor. Finally, unfold the hip and knee joints again and return to the starting position. The 4 exercises are described in Table 1.

Apply sEMG to the four muscle groups under the above conditions to compare the activity of each muscle with maximal voluntary isometric contraction (MVIC) and compare the differences in activity of the lower extremity muscles due to instability.

Table 1. Type of exercise.

\begin{tabular}{ll}
\hline Type of exercise & Type of instability \\
\hline Lunge 1: Flat lunge exercise & none \\
Lunge 2: Lunge exercise on TOGU & Bottom-up approach \\
Lunge 3: Olympicbar + lunge on TOGU & Bottom-up approach \\
Lunge 4: Surge + lunge on TOGU & Top-down approach \\
\hline
\end{tabular}




\section{2) sEMG measurement}

To compare muscle activity, an eight-channel radio surface muscle conductor WEMG-8 (LXM5308, LAXTHA, Korea) was used and converted into digital signals using Ag/AgCl electrodes, and surface muscle conduction analogue signals from each of the four channels collected were processed by telescan software (LAXTHA, Korea) using personal laptops. Before applying electrodes, they were sterilized with alcohol to minimize errors caused by skin resistance. In addition, hair was removed from the applying spot to reduce skin's resistance to muscle conduction signals. The surface electrode is attached to the center of the muscle, taking into account the change in position of the muscle belly as the muscle contracts. ${ }^{12}$ Electrode attaching locations are $2.5 \mathrm{~cm}$ from the middle in highest of iliac crest in muscle gluteus medius, vatus medialis was placed over the muscle belly approximately $4 \mathrm{~cm}$ superior to and $3 \mathrm{~cm}$ medial to the superomedial patella border, and orientated $55^{\circ}$ to the vertical, a point 3 $\mathrm{cm}$ above the knee bone, gastrocnemeus is $2 \mathrm{~cm}$ interval from the centerline behind the calf and below the knee, and tibialis anterior is $2 \mathrm{~cm}$ intervals to the muscle belly at $1 / 3$ of the calf bone and inner peach bone. ${ }^{15,16}$

The muscle conduction signal was observed by designating a channel corresponding to the muscles connected to each cable in the laptop connected to the muscle conduction equipment, the sampling rate of the signals collected through the electrodes was set at 1,024 $\mathrm{Hz}$, the band pass filter was set at 10-500 Hz, and the notch filter was filtered to eliminate noise at $60 \mathrm{~Hz}$. All collected muscle conduction signals processed as the root mean square (RMS) to quantify.

To prevent data errors due to differences in muscle strength of each participants used the MVIC, the average muscle conduction signal was normalized to the MVIC for the remaining 3 seconds except the first and last 1 second, after measuring for 5 seconds during maximum isometric contractions for quantitative comparison analysis. The unit of measure for muscle conduction is $\mu \mathrm{V}$.

\section{3) Statistical analysis}

The mean values of measured data were used in this study, and the data processing collected was calculated and compared with the mean and standard deviations for each variable using the commercial statistics program, SPSS ver 22.0 (SPSS Inc., Chicago, IL, USA). The general characteristics of participants were analyzed using technical statistics. The Shapiro-Wilk test was used for a normal distribution. One-way repeated ANOVA was used to analyze data showing differences in activity of the lower extremities, bonferroni adjustment was used as a post-hoc test, and the statistical significance level was $\alpha=0.05$.

\section{RESULTS}

\section{Comparison of muscle activity among exercise groups according to exercise}

While participants were doing 4 types of lunge exercises, there was a difference in muscle activity according to exercise method $(\mathrm{p}<$ 0.05)(Table 2). Post-test results to see the difference in muscle activity between each movement showed that Lunge 4 showed higher activity in all muscles, and the calf muscles were more active than when Lunge 1 performed Lunge 2. The effect of weight and instability caused by olympic-bar and Surge was identified by the muscle conduction value of Lunge 1 is higher than Lunge 3 and Lunge 4. Also, muscle vastus medialis showed the highest muscle activity of Lunge 2 (Table 2)(Figure 1). ${ }^{18-20}$

\section{DISCUSSION}

The purpose of this study is to propose an unstable resistance device that is most effective in the lunge to balance and strengthen the muscles of the lower extremities of normal adult males. The results of the preceding study showed needs to explore again for the pur-

Table 2. Comparison of each muscles EMG during to lunge exercise.

(Unit: \%MVIC)

\begin{tabular}{|c|c|c|c|c|c|c|c|}
\hline & Lunge 1 (a) & Lunge 2 (b) & Lunge 3 (c) & Lunge $4(d)$ & $\mathrm{F}$ & $\mathrm{p}$ & Post-hoc \\
\hline G.Med & $17.36 \pm 6.49$ & $22 \pm 9.01$ & $27.49 \pm 9.93$ & $38.96 \pm 8.25$ & 70.283 & $0.000^{*}$ & $a, b, c<d$ \\
\hline VM & $8.81 \pm 4.04$ & $23 \pm 9.2$ & $12.6 \pm 7.05$ & $16.92 \pm 6.61$ & 43.341 & $0.000^{*}$ & $\begin{array}{c}a, c<b \\
a<d\end{array}$ \\
\hline GCM & $19 \pm 8.81$ & $10.9 \pm 5.12$ & $31 \pm 9.93$ & $43 \pm 9.45$ & 71.801 & $0.001^{*}$ & $b<a<c<d$ \\
\hline TA & $22.04 \pm 7.45$ & $31.73 \pm 8.67$ & $44.11 \pm 9.63$ & $68.21 \pm 5.65$ & 170.287 & $0.000^{*}$ & $a<b<c<d$ \\
\hline
\end{tabular}

G.Med: gluteus medius, VM: vastus medialis, GCM: gastrocnemius, TA: tibialis anterior. ${ }^{*} p<0.05$. 


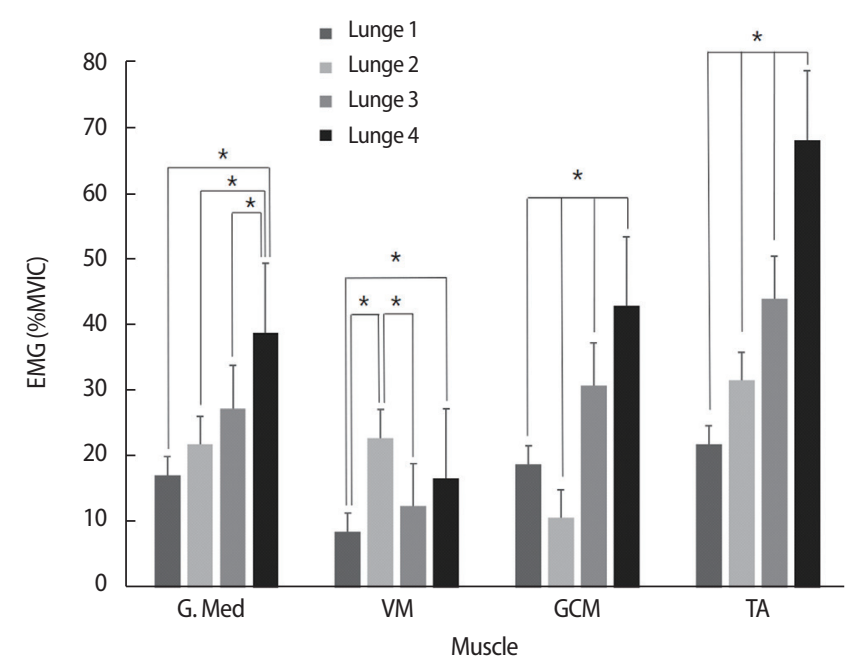

Figure 1. Comparison of each muscles EMG during to lunge exercise. G.Med: gluteus medius, VM: vastus medialis, GCM: gastrocnemeus, TA: tibialis anterior. ${ }^{*} p<0.05$.

pose of the instability device. ${ }^{19}$ It is expected that these various devices will be more effective if they are carried out in accordance with their purpose in carrying out the lunge exercises. The lunge requires more left and right movement and balance than the squats, since weight is applied to the legs on the floor, storing the propulsion in elastic energy, and then converting it back into a thrust that returns to its place. And also requires continuous and dynamic control of the lower extremities to maintain adequate stability because it is applied on narrow floors. ${ }^{7,8}$ Therefore, it has been recommended as a suitable exercise to apply a variety of instability devices. Also, lunge exercises can be easily performed by anyone, even if there are no special devices or assistants in narrow areas and spaces, and as one of the closed chain exercises for special functional needs, they are beneficial for the activity and training of muscle tissue. ${ }^{22}$ Adding purpose-appropriate instability devices to this can increase the effectiveness of exercise and is also a training method that can be used properly in the competitive environment of professional athletes. ${ }^{12}$

In this study, the following four motion muscles were compared and analyzed. Flat lunge exercise (Lunge 1), lunge exercise on TOGU (Lunge 2), Olympic bar + lunge on TOGU (Lunge 3), surge + lunge on TOGU (Lunge 4). EMG was attached to the 4 target muscles, muscle gluteus medius, muscle vastus medialis, gastrocnemius muscle and anterior to measure the activity of the muscles and compared by MVIC. There was a significant difference in the activity of muscles in each of the action $(\mathrm{p}<0.05)$.
Although Lunge 1 had lower muscle activity than Lunge 2, the muscle activity of gastrocnemius muscle in Lunge 1 was rather high. This means that the ground reaction force of Lunge 1 has become more active on the dominant foot located in the front. ${ }^{17}$ The activity of gastrocnemius muscle for Lunge 3 and Lunge 4 was higher than that for Lunge 1. This means that the weight of the Olympic-bar and the weight of the surge, the ground reaction force and the top-down method contributed to the instability.

Nairn et al. ${ }^{1}$ measured muscle activity using three instability tools (bar, bosu, water tube) during squat exercise. In the exercise on bosu, a condition similar to lunge 2, the muscle activity of gastrocnemeus was higher than that of all other movements. However, Lunge 2 showed vastus medialis higher than all other movements. This means that muscle vastus medialis contributes to the stability of the knee compared to Lunge 1. And higher than the types using Olympic-bar and Surge show that more weight loads and instability have made them more active as a reward for other muscles. ${ }^{17}$ Thus, to selectively strengthen muscle vastus medialis, it is more effective to exclude the instability and weight load of top-down instability or to exclude only the weight load when there is bottom-up instability. This is consistent with the results of a prior study that, if instability increases above a certain level, the activation value of the muscle quadriceps femoris are reduced. ${ }^{18-20}$

All 4 muscle activity levels in Lunge 3 were lower than in Lunge 4. Lunge 4 is a combination of bottom-up instability and top-down instability that affects lower extremity muscle activity than lunge 3 performed with bottom-up instability. The use of bottom-up instability for the purpose is considered to be an effective means of intervention for tasks that consider the characteristics of the patient's individual, as well as the lunge exercises.

The generally application of the results of this study has the following limitations.First, this study limited the participants to only young adults in 20s, so clinical application to patients is too much. In addition, the lasting effects of the lunge were not known. However, this study will provide clinically sufficient value for application of purpose-appropriate instability resistance devices by presenting the results of the lunge exercise methods performed in various environments. In future studies, I wish to complement the limitations of this study, including the identification of various exercise movements, various participants, and continuous motion effects, so, can present lunge methods that can be closer to generalization. 


\section{CONCLUSION}

The purpose of this study was to compare the activity of lower extremity muscles among 20 healthy adult males in terms of instability, and to identify the device for instability that can be effectively applied.

Thus, in this study, 4 lunge actions (Lunge 1, 2, 3,4) were performed using sEMG devices and instability devices. There was a total of 4 target lower extremity muscles, muscle gluteus medius, muscle vastus medialis, gastrocnemius muscle and anterior, and activity was measured and compared through surface muscle conduction tests.

The results of this study showed a significant difference in muscle activity depending on the performance of each action $(p<0.05)$. The effect of top-down method on lower limb muscles was checked. In conclusion, top-down methods, like Bottom-up devices, can be used as training and intervention methods to provide an environment suitable for athletes' rehabilitation programs or athletic purposes, if used in accordance with their situations and purposes.

\section{REFERENCES}

1. Nairn BC, Sutherland CA, Drake JD. Motion and muscle activity are affected by instability location during a squat exercise. J Strength Cond Res. 2017;31(3):677-85.

2. Behm DG, Anderson K, Curnew RS. Muscle force and activation under stable and unstable conditions. J Strength Cond Res. 2002;16(3):416-22.

3. Park J, Kim Y. A study on changes in knee joint loading during stair gait with unstable shoes. J Kor Phys Ther. 2014;26.

4. Yeo S, Yoon S, Park G. A comparison muscle activity of lower limb muscle for men in their 20s during lunge motion and kettlebell swing. Korean J Sport Sci. 2016;25(25):1219-6.

5. Fulkerson JP. Diagnosis and treatment of patients with patellofemoral pain. Am J Sports Med. 2002;30(3):447-56.

6. Cook G. Movement: functional movement systems: screening, assessment, corrective strategies. On Target Publications, 2011.

7. Cronin J, McNair P, Marshall R. Lunge performance and its determi- nants. J Sports Sci. 2003;21(1):49-57.

8. Keynes M. Level 1: Assistant coach training manual badminton association of england. 2006.

9. Kuntze G, Mansfield N, Sellers W. A biomechanical analysis of common lunge tasks in badminton. J Sports Sci. 2010;28(2):183-91.

10. Moreside JM, Vera-Garcia FJ, McGill SM. Trunk muscle activation patterns, lumbar compressive forces, and spine stability when using the bodyblade, Phys Ther, 2007;87(2):153-63.

11. Kim Y, Kim Y. Unilateral performance comparison for taekwondo kicks between dominant leg and non-dominant leg. Korean Journal of Sport Biomechanics. 2010;20(2):183-9.

12. Behm DG, Anderson KG. The role of instability with resistance training. J Strength Cond Res 2006;20(3):716.

13. Palluel E, Ceyte H, Olivier I et al. Anticipatory postural adjustments associated with a forward leg raising in children: effects of age, segmental acceleration and sensory context. Clin Neurophysiol. 2008;119(11): 2546-54.

14. Kim KS, Shin HK. The effects of the sling strap height on trunk and hip muscle activation during the bridging exercise with sling. J Kor Phys Ther. 2016;28(1):59-63.

15. Lyons K, Perry J, Gronley JK et al. Timing and relative intensity of hip extensor and abductor muscle action during level and stair ambulation: an EMG study. PhAys Ther. 1983;63(10):1597-605.

16. Kim J. Effects of EMG-biofeedback using closed kinetic chain exercise on Q-angle and quadriceps muscle activation in patellofemoral pain syndrome. J Kor Phys Ther. 2016;28(2):65-70.

17. Hatfield DL, Kraemer WJ, Spiering BA et al. The impact of velocity of movement on performance factors in resistance exercise. J Strength Cond Res. 2006;20(4):760.

18. Drinkwater EJ, Pritchett EJ, Behm DG. Effect of instability and resistance on unintentional squat-lifting kinetics. Int J Sports Physiol Perform. 2007;2(4):400-13.

19. Goodman CA, Pearce AJ, Nicholes CJ et al. No difference in 1RM strength and muscle activation during the barbell chest press on a stable and unstable surface. J Strength Cond Res. 2008;22(1):88-94.

20. McBride JM, Larkin TR, Dayne AM et al. Effect of absolute and relative loading on muscle activity during stable and unstable squatting. Int J Sports Physiol Perform. 2010;5(2):177-83.

21. Drake, JDM, Fischer, et al. Do exercise balls provide a training advantage for trunk extensor exercise? A biomechanical evaluation. J Manipulative Physiol Ther 29: 254-362, 2006.

22. Kisner C, Colby LA, Borstad J, Therapeutic exercise: Foundations and techniques. Philadelphia, Fa Davis. 